\section{A personal tribute to Ralph Kirsch}

To the Editor: The last few months have been awkward for a few reasons. The first was that Ralph's illness and subsequent passing seemed so sudden. The second was that when one is not close family appropriate behaviour is elusive, and finally because there are things I felt were left unsaid.

Ralph's many academic and organisational achievements have been eloquently told by many and are not the subject of this personal account. Let me explain. When I first met Ralph in the mid 1990s, he struck me as a talkative, overly nice, trying-to-make-up-for-apartheid type of man. Our family later moved to Oxford, and one day Ralph and Beverley happened to pass through. Amidst the pleasantries, I was struck by how much attention they both gave to our children - then 7 and 5 . Ralph established that what the children missed most about Cape Town was playing in the swimming pool. By the end of the visit, the kids had an open invitation to swim in his pool. After 2 years of seemingly unending English winters we visited Cape Town, arriving on a warm summer's day. Needless to say, we were made to deliver on the promise and spent far too many hours in the blazing sun.

A few years after our final return home, I sat for my final specialist examinations of the College of Dermatologists. I hate to admit that I failed the examination. Many reading this will have no idea of what that feels like: it's like a gnawing pain that eats one inside, a pain so deep that it threatens to overwhelm. Words of comfort came from many sides, including my favourite which I still use to this day: 'When writing an undergraduate exam, you've passed unless you fail yourself; for specialist exams, you've failed until you pass yourself.' It makes sense, as supposedly the buck stops with specialists. In the midst of this dark cloud, Ralph phoned unexpectedly one evening. He uncharacteristically said only a few words, something like, 'Nonhlanhla, I know this is a difficult time, but I just want you to know that I also had to repeat my FCP examination'. That was it! I was to turn those words round and round in my mind for many months. He was a busy Head of Department who did something he did not have to do, and that was completely unexpected. And for me it was timely and crucial encouragement.

My specialist training left me with a deep desire to explore scientifically unanswered questions on the causes of and treatments for alopecia. I enrolled to do a PhD in Public Health at UCT, having naïvely overestimated the attractiveness of hair loss to potential research funders. When I'd exhausted my options, I went to Ralph who listened and encouraged me to pursue this unlikely research subject in spite of sceptical smiles and sometimes open criticism. He advised on various options and I was able to do the first 2 years full-time through funding from the Carnegie Fund. The rest, as they say, is history! I have no doubt that I would have completed the PhD ... eventually, but it would have been much harder and taken much longer doing it part-time.

What do I now think of Ralph Kirsch? He was a kind man, who cared about apparently insignificant things like organising a swimming date for sun-starved children. He went beyond expectation and encouraged the best in me. He was my hero. Hamba kahle qhawe! Akuhlanga lungehlanga, Beverley nabantwana.

\section{Nonhlanhla P Khumalo}

Associate Professor

Division of Dermatology

Groote Schuur Hospital and Red Cross Children's Hospital

University of Cape Town

n.khumalo@uct.ac.za

\section{Sudden death on an aeroplane}

To the Editor: I was much interested in the report by Tiemensma et al. ${ }^{1}$ Considering the extent of the tension pneumothorax as seen in Fig. 1, one would have thought that the doctors on board should have diagnosed it - was the clinical examination done properly? Was the position of the trachea palpated, the thoracic cage percussed, auscultation of lung and heart sounds done?

With the wonderful and rapid progression of technology and sophisticated diagnostic facilities, it seems that the importance of a proper and detailed medical history, and the value of a complete, meticulous and careful physical examination, are no longer appreciated.

My impression is that the subject is neglected in present-day teaching of medical students. Too time consuming? Life too hurried?

I know of a case in which after a (normal) computed tomography brain scan in a stuporous patient, the correct diagnosis of diabetes was eventually made. I can cite several similar examples.

Patients also constantly complain that their doctors appear so busy that there is no time for questions or explanations.

My 'window of medical experience' over several decades convinces me that there is still a place for a basic down-to-earth clinical approach. Many doctors are going to work far from tertiary care centres.

\section{J G Prinsloo \\ Emeritus Professor of Paediatrics \\ University of Pretoria \\ ida.vanbiljon@up.ac.za \\ 1. Tiemensma M, Buys P, Wadee SA. Sudden death on an aeroplane. S Afr Med J 2010; 100(3): 148-149.}

\section{Pathology request guidelines: Haematology questioned}

To the Editor: We refer to the Haematology section of the article by Stanfliet, King and Pillay. ${ }^{1}$

The assessment of iron deficiency anemia (IDA) is incomplete without demonstrating iron-deficient erythropoiesis, by looking at the red cell indices (mean corpuscular volume, mean cell haemoglobin and mean cell haemoglobin concentration ( $\mathrm{MCV}, \mathrm{MCH}$ and $\mathrm{MCHC}))^{2,3} \mathrm{~A}$ serum ferritin is mandatory if the indices suggest microcytic hypochromic anaemia (MHA). A low ferritin confirms IDA, but a normal ferritin does not exclude iron deficiency. A normal 
or raised ferritin may also indicate another cause of MHA, such as anaemia of chronic disorder (of which HIV and TB are common causes locally). ${ }^{2-4}$ Therefore, such an over-simplified approach will misdiagnose or over-diagnose IDA.

A trial of iron without confirmation of IDA has been proposed, but only for patients who are regarded as low-risk. Included in this group would be premenopausal women and young individuals during growth spurts, especially in areas known to have a high prevalence of IDA. Even in this group, a trial of therapy with $200 \mathrm{mg}$ of ferrous iron is more correctly recommended for 3 weeks prior to review for response, as opposed to the 2 months, as suggested by the authors. ${ }^{3}$ A positive response is present when the haemoglobin concentration $(\mathrm{Hb})$ has increased by $2 \mathrm{~g} / \mathrm{dl}$ after 3 weeks of therapy. This confirms the diagnosis of IDA, the cause of which still needs to be ascertained. Some studies have shown a statistically significant prevalence of dual pathology in this group of patients. ${ }^{4}$ In a prospective study of 187 premenopausal patients with IDA, menorrhagia was present in $67.4 \%$. A possible gastro-intestinal (GI) cause of IDA was found in $57.2 \%$ and $43 \%$ for the upper and lower GI tract respectively $(p<0.04$ and 0.002$)$. This would easily be missed if therapy is given without questioning the need to investigate for a possible cause. The main risk factor for the presence of likely GI causes in this study was the presence of upper GI symptoms (odds ratio 5.2, 95\% confidence interval $1.6-16.4, p=0.005$ ). ${ }^{4}$ Moreover, in high-risk patients (such as the elderly), a trial of iron without establishing a diagnosis of the underlying IDA will result in unnecessary delays and is potentially hazardous to the patient.

Regarding haemoglobin determinations, there has been significant disparity between using the Spencer Haemoglobinometer readings and the standard laboratory readings reported in the study. Observers' $\mathrm{Hb}$ estimations, using the Hbmeter, ranged around $7.2 \mathrm{~g} / \mathrm{dl}$ and $7.8 \mathrm{~g} / \mathrm{dl}$ for a laboratory $\mathrm{Hb}$ of $5.9 \mathrm{~g} / \mathrm{dl}$, questioning the accuracy of an instrument on which therapeutic decisions may be made. ${ }^{5}$

\section{K Moeng}

\section{A D Mahomed}

Division of Medical Gastroenterology

Department of Internal Medicine

University of the Witwatersrand

Johannesburg

adam.mahomed@wits.ac.za

\section{Patel}

Division of Haematology

Department of Internal Medicine

University of the Witwatersand

1. Stanfliet JC, King JA, Pillay TS. Inadequacy of primary health care test request guidelines-Lack of an evidence base. S Afr Med J 2009; 99: 569-571.

2. Cook JD. Diagnosis and management of iron-deficiency anaemia. Best Prac Res Clin Haemato 2005; 18(2): 319-332.

3. Frewin R, Henson A, Provan D. ABC of clinical haematology: iron deficiency anaemia. BMJ 1997; 314: 360-363.

4. Smellie WSA, Forth J, Coleman JJ, et al. Best practice of primary care pathology: Review 3. J Clin Pathol 2006; 59: 781-789.

5. Vannella L, Aloe Spiriti MA, Cozza G. Benefit of concomitant gastrointestinal and gynaecological evaluation in pre-menopausal women with iron deficiency anaemia. Aliment gynaecological evaluation in pre-
Pharmacol Ther 2008; 28: 422-430.

6. Discombe G, Goddard AJ, Hill ID. The accuracy of haemoglobin determinations with the Spencer Haemoglobinometer. J Coll Gen Pract 1966; 12(2): 251-252.
Stanfliet, King and Pillay respond: Linegar et al. investigated the Buffalo haemoglobinometer. ${ }^{1}$ The guidelines recommend the use of the Spencer haemoglobinometer from American Optical. However, the design and principles of both instruments are similar, and identical considerations apply.

The performance of several portable haemoglobinometers is not without problems. ${ }^{2}$ The study cited by Moeng et al. quoting a CV of $3 \%$ obtained for the Spencer haemoglobinometer, was not conducted as would be the case today. No correlation co-efficient is stated, and it is not possible to comment on the appropriateness of the use of the haemoglobinometer, although they recommend it for general practice. ${ }^{3}$

There is no consensus on the investigation and management of iron deficiency anaemia (IDA). ${ }^{2}$ The gold standard for the diagnosis of iron deficiency is bone marrow aspirate stained for iron, and no single biomarker can serve as a proxy. ${ }^{2}$ We agree that a blind trial of iron supplementation is inappropriate purely on the basis of a portable haemoglobinometer, given the poor correlation of some devices in the primary health care setting, with some workers reporting a correlation factor of 0.61 against a formal haemoglobinometer, but that it serves as a screening test for anaemia in a primary health care setting. ${ }^{4}$ Nevertheless, Adamson et al. reported on children in the primary health care setting, where they reserved the measurement of serum ferritin for those with severe anaemia $(\mathrm{Hb}<9.5 \mathrm{~g} / \mathrm{dl})$. Those with milder anaemia purely diagnosed by a handheld haemoglobinometer, and no other indices, were treated with dietary advice and community follow-up. ${ }^{5}$

We suggest the use of further markers in addition to the hand-held haemoglobinometer to confirm the anaemia and to identify its likely cause. ${ }^{2,6}$ Ferritin and soluble transferrin receptors are probably the best biomarkers for the assessment of iron status in the absence of inflammation, but interpretation in the presence of inflammation and malignancy is difficult. ${ }^{2,6-8}$ Some authors only measure serum ferritin if the markers of microcytic anaemia are not consistent with IDA. ${ }^{6}$ Spuriously elevated serum ferritin levels can also be seen in hepatocellular damage and in situations such as non-steroidal anti-inflammatory agent-induced upper gastrointestinal bleed. ${ }^{6}$ Work in the interpretation of ferritin in the setting of inflammation and to improve guidelines is urgently needed. ${ }^{7,8}$

A further consideration is whether a serum ferritin is logistically possible or economically feasible in the primary health care setting in all patients in our resource-constrained settings. Further work is required to expand the current evidence base.

Vannella et al. reported that $93.6 \%$ of enrolled patients were refractory to oral iron supplementation and were referred for investigation. ${ }^{9}$ Their study population had a high incidence of coeliac disease and Helicobacter infection, and they suggested non-invasive serological tests to better decide who might benefit from gastroscopy. ${ }^{9}$ It therefore appears that referral for further investigation and delineation of the cause of IDA in 'low-risk' patients should be reserved for those who prove refractory to a trial of oral iron treatment.

With regard to assessing the response to oral iron therapy, an increase in $\mathrm{Hb}$ of $2 \mathrm{~g} / \mathrm{dl}$ in 3 weeks is historically considered as 